types of organelle are potentially autonomous, since they each contain DNA, DNA polymerase, RNA polymerase, and a protein-synthesising system, but all the evidence suggests that most organelle proteins are encoded in the nucleus and are synthesised by cytoplasmic ribosomes. The major problems discussed were the identification of the genes in the organelle DNA and of the proteins synthesised by organelle ribosomes.

The interactions between nuclear and organelle genomes in the synthesis of organelle proteins are complex, but are yielding to modern techniques. Two general principles are emerging: (1) one of the functions of the organelle DNA is to encode some subunits of multisubunit organelle proteins, the remaining subunits being encoded in nuclear DNA; (2) those proiein subunits encoded in organelle DNA are synthesised by organelle ribosomes, whereas protein subunits encoded in nuclear DNA are synthesised on cytoplasmic ribosomes. For example, D. E. Griffiths (University of Warwick) reviewed data on yeast mitochondrial ATP-synthetase, which is composed of ten subunits; six of these are nuclear coded and made on cytoplasmic ribosomes, whereas the other four are made by mitochondrial ribosomes and are presumed to be encoded in mitochondrial DNA. The major chloroplast protein (fraction I protein or ribulose diphosphate carboxylase) is composed of large and small subunits. R. J. Ellis (University of Warwick) described the synthesis of the large, but not the small subunit, by isolated intact chloroplasts and by free chloroplast ribosomes. Tryptic peptide analysis of mutant tobacco plants has shown that the large subunit is encoded in chloroplast DNA whereas the small subunit is encoded in nuclear DNA. The emphasis of work in this area is now shifting away from the identification of products of the different genomes towards a study of the regulatory mechanisms existing between them.

Elegant studies of mitochondrial DNA isolated from cytoplasmic petite mutants of yeast were reported by $\mathrm{P}$. Slonimski (CNRS, Gif-sur-Yvette); this DNA is derived from wild type DNA by large deletions which are replaced by reiterations of the non-deleted segments. The resulting gene redundancy can be detected by electron microscopic studies of half-denatured molecules. Stable petite mutants in which different parts of the mitochondrial DNA are repeated and amplified up to 100 times can be isolated, thus opening the way for the selective purification of different mitochondrial genes. Such studies on chloroplast DNA are much less advanced because of the difficulty of inducing mutations in the DNA of this type of organelle; R. Hagemann
(Martin Luther University, Halle) reported that some naturally-occurring chloroplast mutations in higher plants are correlated with the absence of spe. cific thylakoid proteins associated with photosystems I and II, and other mutations result in a loss of chloroplast ribosomes.

All of the products of protein synthesis by mitochondrial ribosomes, and most of those by chloroplast ribosomes, are membrane-bound. This raises severe problems of purification and isolation, but these problems are being steadily overcome. In a tour de force, $\mathbf{R}$. $\mathrm{O}$. Poyton (University of Connecticut) reported the isolation in milligram quantities of each of the seven subunits of yeast cytochrome oxidase. Four of these are made by cytoplasmic ribosomes and are water soluble when isolated, but the other three are made by mitochondrial ribosomes and remain insoluble. The three insoluble subunits are synthesised by isolated mitochondria, provided that oxygen is present; in anaerobic conditions, only one subunit is made. The mechanism of this interesting effect is unknown, but could reflect a specific effect of oxygen on mitochondrial transcription or translation.

On the second day, further work in the area of organelle biogenesis was discussed. G. S. P. Groot (University of
Amsterdam) suggested from an analysis of base sequence homology in nuclear and mitochondrial DNAs of different yeasts that mitochondrial DNA evolves faster than nuclear DNA, and L. A. Grivell (University of Amsterdam) reported that yeast mitochondrial ribosomal RNA is extraordinary in its complete lack of ribose methyl groups and a very low level of base methylation compared with other ribosomal RNAs. A contribution on chloroplast RNA by M. R. Hartley (University of Warwick) was notable, in that it reported, for the first time, the detection of a specific messenger RNA from a plant source (other than a viral messenger). This messenger is for the large subunit of fraction I protein which can be correctily translated by a cell-free protein synthesising system from Escherichia coli. Studies of specific messenger RNAs from plants have lagged far behind those from animals, so this advance should open up a new area of plant molecular research.

Outstanding problems that were raised, but not resolved, were the function of the genes in chloroplast DNA, $90 \%$ of which are still unidentified, and the mechanism of the uptake of specific proteins into and across the outer membranes of mitochondria and chloroplasts.

\title{
Neural attenuation in echolocating bats
}

\author{
from our Animal Behaviour Correspondent
}

THE sonar system possessed by many bats enables them to perform such feats as flying unhindered through an array of fine wires and selecting one insect from a swarm of many. The echoes from surrounding objects must be perceived a few milliseconds after the bats have emitted an extremely intense outgoing sound pulse (often several thousand dynes per $\mathrm{cm}^{2}$ ). Somehow the bats' ears must be protected from their own cries and yet still be sensitive enough to pick up the much fainter returning echoes. There are at least two mechanisms for accomplishing this. The muscles of the middle ear contract synchronously with vocalisation (they can contract and relax again up to 60 times $s^{-1}$ on some species) and this has the effect of attenuating selfstimulation.

In addition to this peripheral mechanism, there are neural mechanisms of attenuation within the auditory pathways. Suga and Schlegel (Science, 177, 82; 1972) made tape recordings of the calls of vespertilionid bats and recorded the response of a bat's auditory nerve both to this tape recording and to the same call when made by the bat itself. Although the response of the primary auditory nerves was virtually the same to both sorts of sound, the inferior colliculus (part of the mid-brain concerned with hearing) showed much less response to the live than to the tape-recorded sound. Attenuation, by some $25 \mathrm{~dB}$, to the bat's own voice was occurring somewhere between the auditory nerve and this part of the brain.

Now Suga and Shimozawa (Science, 183, 1211; 1974) have pinpointed the exact site of this neural attenuation. By recording from various nuclei along the auditory pathway when bats of the genus Myotis were either vocalising themselves or listening to tape recordings, they found that neural attenuation occurs in the nucleus of the lateral lemniscus. They point out that although such mechanisms are of obvious importance for bats with their special problems in echolocation, similar muscular and neural attenuation mechanisms may also operate in human speech, since our vocalisations rarely appear disturbingly loud. 